Vietnam Journal of Mechanics, VAST, Vol.40, No. 2 (2018), pp. $121-140$

DOI:10.15625/0866-7136/10503

\title{
FREE VIBRATION ANALYSIS OF 2-D FGM BEAMS IN THERMAL ENVIRONMENT BASED ON A NEW THIRD-ORDER SHEAR DEFORMATION THEORY
}

\author{
Tran Thi Thom ${ }^{1,2}$, Nguyen Dinh Kien ${ }^{1,2, *}$ \\ ${ }^{1}$ Institute of Mechanics, VAST, Hanoi, Vietnam \\ ${ }^{2}$ Graduate University of Science and Technology, VAST, Hanoi, Viet Nam \\ *E-mail: ndkien@imech.vast.vn \\ Received July 10, 2017
}

\begin{abstract}
Free vibration analysis of two-directional functionally graded material (2-D FGM) beams in thermal environment based on a new third-order shear deformation theory is presented. The material properties are assumed to be graded in both the thickness and longitudinal directions by a power law distribution, and they are considered to be temperature-dependent. Equations of motion, in which the shear rotation rather than the cross-sectional rotation is considered to be an independent variable, are constructed from Hamilton's principle. A finite element formulation is derived and employed to compute the vibration characteristics of the beams. The numerical results reveal that the developed formulation is accurate, and it is capable to give accurate natural frequencies by using a small number of elements. A parametric study is carried out to highlight the effects of material composition, temperature rise on the vibration characteristics of the beams.
\end{abstract}

Keywords: 2-D FGM beam, temperature-dependent properties, new third-order shear deformation theory, shear rotation, free vibration analysis.

\section{INTRODUCTION}

Beams made of functionally graded materials (FGMs) with excellent thermal resistance are increasingly used as structural components in aircrafts and space vehicles where the effect of temperature is often concerned. Investigations on the static and dynamic behavior of FGM beams are extensively reported in the literature, contributions that are most relevant to the topic of the present work are briefly discussed below.

Chakraborty et al. [1] formulated a first-order shear deformable beam element for studying the static, free vibration and wave propagation problems of bi-material beams fused with an FGM layer. The beam element taking the uniform temperature rise into consideration is derived by using the exact solution of static equilibrium equations of an FGM segment to interpolate the kinematic variables. The third-order shear deformation beam theory was adopted by Kadoli et al. [2] to develop the stiffness matrix and load

(C) 2018 Vietnam Academy of Science and Technology 
vector for the stress analysis of FGM beams. Pradhan and Murmu [3] utilized the modified differential quadrature method to compute natural frequencies of FGM sandwich beams. Mahi et al. [4] presented an analytical method for examining the effect of the temperature rise and material distribution on the natural frequencies of higher-order shear deformable FGM beams. Based on a new third-order shear deformation theory proposed recently by Shi in [5], Wattanasakulpong et al. [6] studied the thermal buckling and vibration of FGM beams. It has been shown by the authors that the fundamental frequency decreases to zero when the temperature increases towards the critical temperature. Alshorbagy et al. [7] used the traditional two-node beam element to compute the natural frequencies of beams with the material properties being graded in the thickness or longitudinal direction by a power-law distribution. Thai and Vo [8] presented analytical solutions for the bending and free vibration problems of higher-order shear deformation FGM beams. The bending and free vibration problems of FGM beams were also considered by Vo et al. in [9] by using a refined shear deformation beam theory. Nguyen et al. [10] studied the bending and free vibration of axially loaded shear deformable FGM beams in which the transverse shear stiffness is derived from the in-plane stress and the shear correction factor is calculated analytically. Recently, Trinh et al. [11] presented an analytical method for vibration and buckling analysis of the third-order shear deformable FGM beams subjected to mechanical and thermal loads.

In the above-discussed references, the beam material properties are considered to be varied in one spatial direction only. There are practical circumstances, in which the unidirectional FGMs may not be so appropriate to resist multi-directional variations of thermal and mechanical loadings. In this regard, the development of FGMs with effective material properties varying in two or three directions to withstand severe general loadings is of great importance in practice. Investigations on the static and dynamic behavior of beams made of two-directional functionally graded materials (2-D FGMs) have been reported by several researchers in recent years. Based on an analytical method, Wang et al. [12] studied the free vibration of 2-D FGM beams by assuming the material properties to be varied in the thickness by an exponent function and in the longitudinal direction by a power law. It has been shown by the authors that the vibration characteristics are significantly influenced by the longitudinal variation of the material properties, and there is a critical frequency at which the natural frequencies have an abrupt jump. Şimşek [13] studied the vibration of 2-D FGM Timoshenko beams traversed by a moving force by assuming the material properties to be varying in the thickness and longitudinal directions by the exponent functions. The author concluded that the material parameters of the 2-D FGM beams could be tailored to meet the design goal of optimizing the dynamic response. Considering the power-law variations of material properties in both the length and thickness directions, Nguyen et al. [14] presented a finite element procedure for studying the forced vibration of 2-D FGM Timoshenko beams excited by a moving load. Using a quasi-3D shear deformation theory and the symmetric smoothed particle hydrodynamics method, Karamanli [15] examined the static behavior of 2-D FGM beams with power-law variation of the material properties in both the length and thickness of the beams. Recently, Do et al. [16] derived a finite element formulation for analysis of 
2-D FGM plates. The formulation is based on the new third-order shear deformation theory proposed by Shi [5], and the cross-sectional rotation is considered as an independent variable.

The shear deformation beam theories which are enabled to model the effect of slenderness ratio are widely employed in analysis of FGM beams. Although a value of 5/6 is usually chosen for the shear deformation factor in the first-order shear deformation theory, this value may not be appropriate for FGM beams with variation of the material properties in the beam thickness [17]. To avoid using the shear correction factor, a new third-order shear deformation theory, derived from an elasticity formulation rather by the hypothesis of displacements in [5] is employed in the present paper to study free vibration of 2-D FGM beams in thermal environment. The material properties of the beams are assumed to vary in both the thickness and longitudinal directions by a power-law function, and they are considered to be temperature-dependent. Equations of motion in which the shear rotation rather than the cross-sectional rotation is considered as an independent variable are derived from Hamilton's principle, and they are solved by a finite element formulation. The choice of the shear rotation as an independent variable is motivated by the fact that the finite element formulation based on the shear rotation is more efficient than the one based on the cross-sectional rotation $[18,19]$. Using the derived finite element formulation, numerical investigations are carried out to highlight the effects of the material composition and temperature rise on the natural frequencies and vibration modes of the 2-D FGM beams.

\section{2-D FUNCTIONALLY GRADED BEAM}

A 2-D FGM beam with length $L$, width $b$ and height $h$ as shown in Fig. 1 is considered. The Cartesian coordinate system $(x, z)$ in the figure is introduced such that the $x$-axis is on the mid-plane, and the $z$-axis is perpendicular to the mid-plane, and it directs upward.

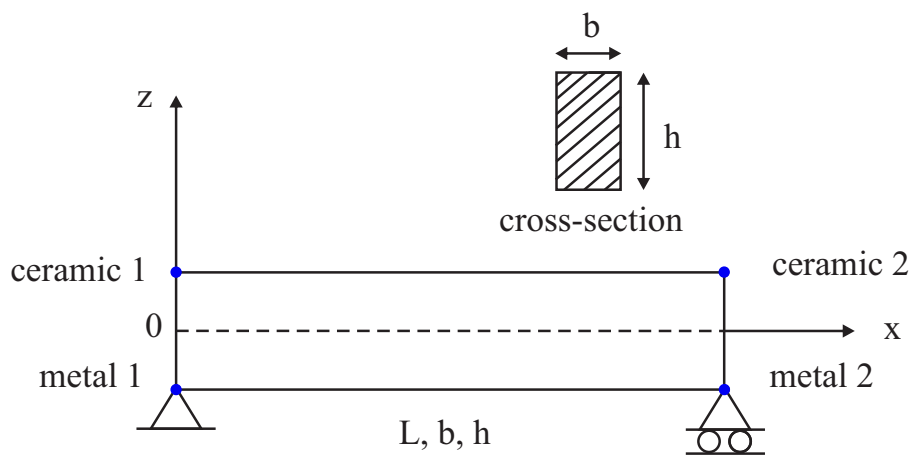

Fig. 1. Geometry and coordinates of 2-D FGM beam

The beam material is assumed to be formed from two ceramics (referred to as ceramic1 and ceramic2) and two metals (referred to as metal1 and metal2) whose volume 
fraction varies in both the thickness and longitudinal directions as

$$
\begin{array}{ll}
V_{c 1}=\left(\frac{z}{h}+\frac{1}{2}\right)^{n_{z}}\left[1-\left(\frac{x}{L}\right)^{n_{x}}\right], & V_{c 2}=\left(\frac{z}{h}+\frac{1}{2}\right)^{n_{z}}\left(\frac{x}{L}\right)^{n_{x}}, \\
V_{m 1}=\left[1-\left(\frac{z}{h}+\frac{1}{2}\right)^{n_{z}}\right]\left[1-\left(\frac{x}{L}\right)^{n_{x}}\right], & V_{m 2}=\left[1-\left(\frac{z}{h}+\frac{1}{2}\right)^{n_{z}}\right]\left(\frac{x}{L}\right)^{n_{x}},
\end{array}
$$

where $n_{z}$ and $n_{x}$ are the grading indexes, which dictate the variation of the constituent materials in the thickness and longitudinal directions, respectively. It can be seen from Eq. (1) that the left and right lower corners of the beam contain only metal1 and metal2, respectively whereas the corresponding upper two corners are, respectively, pure ceramic1 and ceramic2. The variation of the volume fraction of ceramic1 and ceramic2 in the $z$-and $x$-directions according to Eq. (1) is illustrated in Fig. 2 for various values of the grading indexes $n_{z}$ and $n_{x}$.
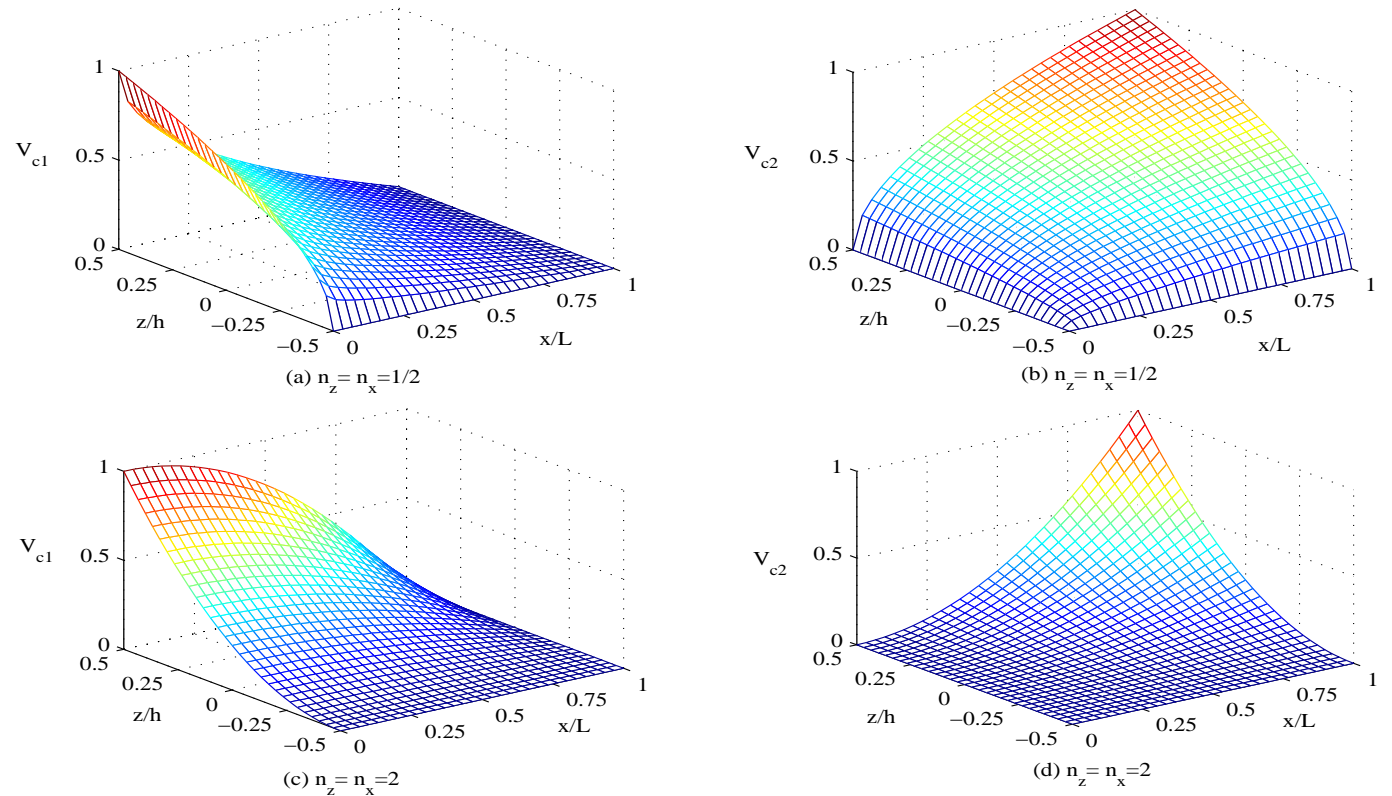

Fig. 2. Variation of volume fraction of ceramics in the thickness and longitudinal directions

The effective material properties $\mathcal{P}$ (such as the elastic modulus and mass density, etc.) are evaluated by Voigt's model according to

$$
\mathcal{P}=V_{c 1} \mathcal{P}_{c 1}+V_{c 2} \mathcal{P}_{c 2}+V_{m 1} \mathcal{P}_{m 1}+V_{m 2} \mathcal{P}_{m 2}
$$

where $\mathcal{P}_{c 1}, \mathcal{P}_{c 2}, \mathcal{P}_{m 1}$ and $\mathcal{P}_{m 2}$ denote the properties of the ceramic1, ceramic2, metal1, and metal2, respectively. Substituting Eq. (1) into Eq. (2), one gets

$$
\begin{aligned}
\mathcal{P}(x, z, T)= & {\left[\left(\mathcal{P}_{c 1}(T)-\mathcal{P}_{m 1}(T)\right)\left(\frac{z}{h}+\frac{1}{2}\right)^{n_{z}}+\mathcal{P}_{m 1}(T)\right]\left[1-\left(\frac{x}{L}\right)^{n_{x}}\right] } \\
& +\left[\left(\mathcal{P}_{c 2}(T)-\mathcal{P}_{m 2}(T)\right)\left(\frac{z}{h}+\frac{1}{2}\right)^{n_{z}}+\mathcal{P}_{m 2}(T)\right]\left(\frac{x}{L}\right)^{n_{x}} .
\end{aligned}
$$


In the above equation, the beam is considered to be in a thermal environment and the material properties depend upon the environment temperature $T$. Noting that the mass density $\rho$ is hardly changed with temperature and it can be considered to be temperatureindependent [6].

One can easily verify that if $n_{x}=0$, Eq. (3) deduces to the well-known expression for the effective material properties of a unidirectional transverse FGM beam made of ceramic2 and metal2. In addition, if ceramic1 and ceramic2 are identical, and metal1 is the same as metal2, Eq. (3) also reduces to the formula for the effective properties of two-phase transversely FG beams. Furthermore, if $n_{z}=0$, Eq. (3) results in the effective material properties of an axially FGM beams formed from ceramic1 and ceramic2.

The material properties are considered to be temperature-dependent, in which a typical material property $\mathcal{P}$ is considered as a function of environment temperature $T(\mathrm{~K})$ as [20]

$$
\mathcal{P}=\mathcal{P}_{0}\left(\mathcal{P}_{-1} T^{-1}+1+\mathcal{P}_{1} T+\mathcal{P}_{2} T^{2}+\mathcal{P}_{3} T^{3}\right),
$$

where $\mathcal{P}_{0}, \mathcal{P}_{-1}, \mathcal{P}_{1}, \mathcal{P}_{2}$ and $\mathcal{P}_{3}$ are the coefficients of temperature and they are unique to the constituent materials, $T=T_{0}+\Delta T$ with $T_{0}=300 \mathrm{~K}$ is reference temperature and $\Delta T$ is the temperature rise. A uniform temperature rise (UTR) is assumed in the present work.

\section{GOVERNING EQUATIONS}

The new third-order shear deformation theory derived from an elasticity formulation, rather by the hypothesis of displacements is employed herewith to establish governing equations of the 2-D FGM beam. The axial and transverse displacements at any point of the beam, $u(x, z, t)$ and $w(x, z, t)$, according to the new theory are of the form [5]

$$
u(x, z, t)=u_{0}(x, t)+\frac{1}{4} z\left(5 \theta+w_{0, x}\right)-\frac{5}{3 h^{2}} z^{3}\left(\theta+w_{0, x}\right), \quad w(x, z, t)=w_{0}(x, t)
$$

where $t$ is the time variable; $u_{0}(x, t)$ and $w_{0}(x, t)$ are, respectively, the axial and transverse displacements of the point on the $x$-axis, and $\theta$ is the cross-sectional rotation.

Using a notation for the transverse shear rotation $\gamma_{0}$, defined as

$$
\gamma_{0}=\theta+w_{0, x}
$$

the axial and transverse displacements in Eq. (5) can be rewritten in the following form

$$
u(x, z, t)=u_{0}(x, t)+z\left(\frac{5}{4} \gamma_{0}-w_{0, x}\right)-\frac{5}{3 h^{2}} z^{3} \gamma_{0}, \quad w(x, z, t)=w_{0}(x, t) .
$$

The axial strain $\left(\varepsilon_{x x}\right)$ and shear strain $\left(\gamma_{x z}\right)$ resulted from Eq. (7) are of the forms

$$
\varepsilon_{x x}=u_{0, x}+z\left(\frac{5}{4} \gamma_{0, x}-w_{0, x x}\right)-\frac{5}{3 h^{2}} z^{3} \gamma_{0, x}, \quad \gamma_{x z}=5\left(\frac{1}{4}-\frac{1}{h^{2}} z^{2}\right) \gamma_{0} .
$$

Based on the assumption of Hooke's law, the constitutive relation for the 2-D FGM beam is as follows

$$
\sigma_{x x}=E(x, z, T) \varepsilon_{x x}, \quad \tau_{x z}=G(x, z, T) \gamma_{x z},
$$

where $E(x, z, T)$ and $G(x, z, T)$ are, respectively, the elastic modulus and shear modulus, which are functions of both the coordinates $x, z$ and they depend on the temperature 
according to Eq. (3); $\sigma_{x x}$ and $\tau_{x z}$ are the axial stress and shear stress, respectively. The elastic strain energy resulted from beam deformation $\left(\mathcal{U}^{B}\right)$, and the kinetic energy $(\mathcal{T})$ of the beam are then given by

$$
\mathcal{U}^{B}=\frac{1}{2} \int_{0}^{L} \int_{A}\left(\sigma_{x x} \varepsilon_{x x}+\tau_{x z} \gamma_{x z}\right) d A d x, \quad \mathcal{T}=\frac{1}{2} \int_{0}^{L} \int_{A} \rho(x, z)\left(\dot{u}^{2}+\dot{w}^{2}\right) d A d x,
$$

where $\rho(x, z)$ and $A=b h$ are, respectively, the mass density and cross-sectional area.

From Eqs. (8) and (9), one can write the elastic strain energy for the beam in the form

$$
\begin{aligned}
\mathcal{U}^{B}= & \frac{1}{2} \int_{0}^{L}\left[A_{11} u_{0, x}^{2}+2 A_{12} u_{0, x}\left(\frac{5}{4} \gamma_{0, x}-w_{0, x x}\right)+A_{22}\left(\frac{5}{4} \gamma_{0, x}-w_{0, x x}\right)^{2}-\frac{10}{3 h^{2}} A_{34} u_{0, x} \gamma_{0, x}\right. \\
& \left.-\frac{10}{3 h^{2}} A_{44} \gamma_{0, x}\left(\frac{5}{4} \gamma_{0, x}-w_{0, x x}\right)+\frac{25}{9 h^{4}} A_{66} \gamma_{0, x}^{2}+25\left(\frac{1}{16} B_{11}-\frac{1}{2 h^{2}} B_{22}+\frac{1}{h^{4}} B_{44}\right) \gamma_{0}^{2}\right] d x,
\end{aligned}
$$

and the kinetic energy resulted from Eq. (7) is as follows

$$
\begin{gathered}
\mathcal{T}=\frac{1}{2} \int_{0}^{L}\left[I_{11}\left(\dot{u}_{0}^{2}+\dot{w}_{0}^{2}\right)+2 I_{12} \dot{u}_{0}\left(\frac{5}{4} \dot{\gamma}_{0}-\dot{w}_{0, x}\right)+I_{22}\left(\frac{5}{4} \dot{\gamma}_{0}-\dot{w}_{0, x}\right)^{2}\right. \\
\left.-\frac{10}{3 h^{2}} I_{34} \dot{u}_{0} \dot{\gamma}_{0}-\frac{10}{3 h^{2}} I_{44} \dot{\gamma}_{0}\left(\frac{5}{4} \dot{\gamma}_{0}-\dot{w}_{0, x}\right)+\frac{25}{9 h^{4}} I_{66} \dot{\gamma}_{0}^{2}\right] d x .
\end{gathered}
$$

The strain energy resulted from the initial stressed due to temperature rise $\left(\mathcal{U}^{T}\right)$ is [4]

$$
\mathcal{U}^{T}=\frac{1}{2} \int_{0}^{L} N_{T} w_{0, x}^{2} d x .
$$

In Eqs. (11), (12) and (13), $A_{11}, A_{12}, A_{22}, A_{34}, A_{44}, A_{66}$ and $B_{11}, B_{22}, B_{44}$ are the beam rigidities, defined as

$$
\begin{aligned}
& \left(A_{11}, A_{12}, A_{22}, A_{34}, A_{44}, A_{66}\right)(x, z, T)=\int_{A} E(x, z, T)\left(1, z, z^{2}, z^{3}, z^{4}, z^{6}\right) d A, \\
& \left(B_{11}, B_{22}, B_{44}\right)(x, z, T)=\int_{A} G(x, z, T)\left(1, z^{2}, z^{4}\right) d A .
\end{aligned}
$$

$I_{11}, I_{12}, I_{22}, I_{34}, I_{44}, I_{66}$ are the mass moments, defined as

$$
\left(I_{11}, I_{12}, I_{22}, I_{34}, I_{44}, I_{66}\right)(x, z)=\int_{A} \rho(x, z)\left(1, z, z^{2}, z^{3}, z^{4}, z^{6}\right) d A
$$

and $N_{T}$ is thermal resultant, which can be expressed as

$$
N_{T}(x, z, T)=-\int_{A} E(x, z, T) \alpha(x, z, T) \Delta T d A,
$$


with $\alpha(x, z, T)$ is the thermal expansion, and $\Delta T$, as above mentioned, is the temperature rise. forms

Substituting Eq. (3) into Eq. (14), one can rewrite the beam rigidities in the following

$$
A_{i j}(x, z, T)=A_{i j}^{c 1 m 1}-\left(A_{i j}^{c 1 m 1}-A_{i j}^{c 2 m 2}\right)\left(\frac{x}{L}\right)^{n_{x}}, B_{i j}(x, z, T)=B_{i j}^{c 1 m 1}-\left(B_{i j}^{c 1 m 1}-B_{i j}^{c 2 m 2}\right)\left(\frac{x}{L}\right)^{n_{x}} .
$$

In the above equation, $A_{i j}^{c 1 m 1}, B_{i j}^{c 1 m 1}$ are the rigidities of the unidirectional transverse FGM beam composed of ceramic1 and metal1; $A_{i j}^{c 2 m 2}, B_{i j}^{c 2 m 2}$ are the rigidities of the transverse FGM beam composed of ceramic2 and metal2. One can see from Eq. (17) that the rigidities of the present 2-D FG beam degenerate to the ones of the unidirectional FGM beam if $n_{x}=0$ or the two ceramics and two metals are identical. Because $A_{i j}^{c 1 m 1}, B_{i j}^{c 1 m 1}, A_{i j}^{c 2 m 2}, B_{i j}^{c 2 m 2}$ are functions of $z$ only, the explicit expressions for the rigidities of the unidirectional transverse FGM beam can easily be obtained [6].

Similarly, the mass moments defined by Eq. (15) can also be rewritten in the forms

$$
I_{i j}(x, z)=I_{i j}^{c 1 m 1}-\left(I_{i j}^{c 1 m 1}-I_{i j}^{c 2 m 2}\right)\left(\frac{x}{L}\right)^{n_{x}} .
$$

Applying Hamilton's principle to Eqs. (11), (12), and (13), one can obtain the following equations of motion for the 2-D FGM beam

$$
\begin{aligned}
& I_{11} \ddot{u}_{0}+I_{12}\left(\frac{5}{4} \ddot{\gamma}_{0}-\ddot{w}_{0, x}\right)-\frac{5}{3 h^{2}} I_{34} \ddot{\gamma}_{0}-\left[A_{11} u_{0, x}+A_{12}\left(\frac{5}{4} \gamma_{0, x}-w_{0, x x}\right)-\frac{5}{3 h^{2}} A_{34} \gamma_{0, x}\right]_{, x}=0, \\
& I_{11} \ddot{w}_{0}+\left[I_{12} \ddot{u}_{0}+I_{22}\left(\frac{5}{4} \ddot{\gamma}_{0}-\ddot{w}_{0, x}\right)-\frac{5}{3 h^{2}} I_{44} \ddot{\gamma}_{0}\right]_{, x}{ }_{,} \\
& -\left[A_{12} u_{0, x}+A_{22}\left(\frac{5}{4} \gamma_{0, x}-w_{0, x x}\right)-\frac{5}{3 h^{2}} A_{44} \gamma_{0, x}\right]_{, x x}-\left(N_{T} w_{0, x}\right)_{, x}=0, \\
& \frac{1}{4} I_{12} \ddot{u}_{0}+\frac{1}{4} I_{22}\left(\frac{5}{4} \ddot{\gamma}_{0}-\ddot{w}_{0, x}\right)-\frac{1}{3 h^{2}} I_{34} \ddot{u}_{0}-\frac{1}{3 h^{2}} I_{44}\left(\frac{5}{2} \ddot{\gamma}_{0}-\ddot{w}_{0, x}\right)+\frac{5}{9 h^{4}} I_{66} \ddot{\gamma}_{0} \\
& -\left[\frac{1}{4} A_{12} u_{0, x}+\frac{1}{4} A_{22}\left(\frac{5}{4} \gamma_{0, x}-w_{0, x x}\right)-\frac{1}{3 h^{2}} A_{34} u_{0, x}-\frac{1}{3 h^{2}} A_{44}\left(\frac{5}{2} \gamma_{0, x}-w_{0, x x}\right)-\frac{5}{9 h^{4}} A_{66} \gamma_{0, x}\right]_{, x} \\
& +5\left(\frac{1}{16} B_{11}-\frac{1}{2 h^{2}} B_{22}+\frac{1}{h^{4}} B_{44}\right) \gamma_{0}=0 .
\end{aligned}
$$

Since the coefficients of the differential equations (19) are functions of $x$, a closedform solution is difficult to derive. Here, a finite element formulation is developed and employed in solving Eq. (19). The beam is assumed to be divided into a number of two-node beam elements with length of $l$. The vector of nodal displacements $(\mathbf{d})$ for the element considering the transverse shear rotation $\gamma_{0}$ as an independent variable contains eight components as

$$
\mathbf{d}=\left\{\begin{array}{lllllllll}
u_{i} & w_{i} & w_{x i} & \gamma_{i} & u_{j} & w_{j} & w_{x j} & \gamma_{j}
\end{array}\right\}^{T}
$$


where $u_{i}, w_{i}, w_{x i}$ and $\gamma_{i}$ are the values of $u_{0}, w_{0}, w_{0, x}$ and $\gamma_{0}$ at the node $i$; and $u_{j}, w_{j}, w_{x j}$ and $\gamma_{j}$ are the corresponding values at the node $j$. In Eq. (20), and hereafter, a superscript ' $T$ ' is used to denote the transpose of a vector or a matrix.

The axial displacement $u_{0}(x, t)$, transverse displacement $w_{0}(x, t)$ and transverse shear deformation $\gamma_{0}(x, t)$ are interpolated from the nodal values according to

$$
u_{0}=\mathbf{N}_{u} \mathbf{d}, w_{0}=\mathbf{N}_{w} \mathbf{d}, \gamma_{0}=\mathbf{N}_{\gamma} \mathbf{d},
$$

where $\mathbf{N}_{u}, \mathbf{N}_{w}$, and $\mathbf{N}_{\gamma}$ denote the matrices of shape functions for $u_{0}, w_{0}$ and $\gamma_{0}$, respectively. In the present work, linear shape functions are used for the axial displacement $u_{0}(x, t)$ and the shear rotation $\gamma_{0}(x, t)$, Hermite shape functions are employed for the transverse displacement $w_{0}(x, t)$ as

- Axial displacement shape functions

$$
N_{u 1}=\frac{l-x}{l}, \quad N_{u 5}=\frac{x}{l}, \quad N_{u 2}=N_{u 3}=N_{u 4}=N_{u 6}=N_{u 7}=N_{u 8}=0 .
$$

- Transverse displacement shape functions

$$
\begin{aligned}
& N_{w 1}=N_{w 4}=N_{w 5}=N_{w 8}=0, \\
& N_{w 2}=1-3\left(\frac{x}{l}\right)^{2}+2\left(\frac{x}{l}\right)^{3}, \quad N_{w 3}=x-2 \frac{x^{2}}{l}+\frac{x^{3}}{l^{2}}, \\
& N_{w 6}=3\left(\frac{x}{l}\right)^{2}-2\left(\frac{x}{l}\right)^{3}, \quad N_{w 7}=-\frac{x^{2}}{l}+\frac{x^{3}}{l^{2}} .
\end{aligned}
$$

- Transverse shear rotation shape functions

$$
N_{\gamma 1}=N_{\gamma 2}=N_{\gamma 3}=N_{\gamma 5}=N_{\gamma 6}=N_{\gamma 7}=0, \quad N_{\gamma 4}=\frac{l-x}{l}, \quad N_{\gamma 8}=\frac{x}{l} .
$$

Using the above interpolation schemes, one can write the strain energy of the beam defined by Eqs. (11) and (13) as

$$
\mathcal{U}=\mathcal{U}^{B}+\mathcal{U}^{T}=\frac{1}{2} \sum^{n e} \mathbf{d}^{T} \mathbf{k} \mathbf{d},
$$

where ne is the total number of the elements, and $\mathbf{k}$ is the element stiffness matrix with the following form

with

$$
\mathbf{k}=\mathbf{k}_{u u}^{11}+\mathbf{k}_{u \gamma}^{12}+\mathbf{k}_{\gamma \gamma}^{22}+\mathbf{k}_{u \gamma}^{34}+\mathbf{k}_{\gamma \gamma}^{44}+\mathbf{k}_{\gamma \gamma}^{66}+\mathbf{k}_{s S}+\mathbf{k}_{T T},
$$

$$
\begin{aligned}
& \mathbf{k}_{u u}^{11}=\int_{0}^{l} \mathbf{N}_{u, x}^{T} A_{11} \mathbf{N}_{u, x} d x, \quad \mathbf{k}_{u \gamma}^{12}=\int_{0}^{l} \mathbf{N}_{u, x}^{T} A_{12}\left(\frac{5}{4} \mathbf{N}_{\gamma, x}-\mathbf{N}_{w, x x}\right) d x, \\
& \mathbf{k}_{\gamma \gamma}^{22}=\int_{0}^{l}\left(\frac{5}{4} \mathbf{N}_{\gamma, x}^{T}-\mathbf{N}_{w, x x}^{T}\right) A_{22}\left(\frac{5}{4} \mathbf{N}_{\gamma, x}-\mathbf{N}_{w, x x}\right) d x, \quad \mathbf{k}_{T T}=\int_{0}^{l} \mathbf{N}_{w, x}^{T} N_{T} \mathbf{N}_{w, x} d x, \\
& \mathbf{k}_{u \gamma}^{34}=-\frac{5}{3 h^{2}} \int_{0}^{l} \mathbf{N}_{u, x}^{T} A_{34} \mathbf{N}_{\gamma, x} d x, \quad \mathbf{k}_{\gamma \gamma}^{44}=-\frac{5}{3 h^{2}} \int_{0}^{l}\left(\frac{5}{4} \mathbf{N}_{\gamma, x}^{T}-\mathbf{N}_{w, x x}^{T}\right) A_{44} \mathbf{N}_{\gamma, x} d x, \\
& \mathbf{k}_{\gamma \gamma}^{66}=\frac{25}{9 h^{4}} \int_{0}^{l} \mathbf{N}_{\gamma, x}^{T} A_{66} \mathbf{N}_{\gamma, x} d x, \quad \mathbf{k}_{s s}=25 \int_{0}^{l} \mathbf{N}_{\gamma}^{T}\left(\frac{1}{16} B_{11}-\frac{1}{2 h^{2}} B_{22}+\frac{1}{h^{4}} B_{44}\right) \mathbf{N}_{\gamma} d x .
\end{aligned}
$$


Similarly, the kinetic energy in Eq. (12) can be rewritten as

$$
\mathcal{T}=\frac{1}{2} \sum^{n e} \dot{\mathbf{d}}^{T} \mathbf{m} \dot{\mathbf{d}}
$$

where

$$
\mathbf{m}=\mathbf{m}_{u u}^{11}+\mathbf{m}_{u \gamma}^{12}+\mathbf{m}_{\gamma \gamma}^{22}+\mathbf{m}_{u \gamma}^{34}+\mathbf{m}_{\gamma \gamma}^{44}+\mathbf{m}_{\gamma \gamma}^{66}+\mathbf{m}_{w w}^{11},
$$

is the element consistent mass matrix, in which

$$
\begin{aligned}
& \mathbf{m}_{u u}^{11}=\int_{0}^{l} \mathbf{N}_{u}^{T} I_{11} \mathbf{N}_{u} d x, \mathbf{m}_{w w}^{11}=\int_{0}^{l} \mathbf{N}_{w}^{T} I_{11} \mathbf{N}_{w} d x, \mathbf{m}_{u \gamma}^{12}=\int_{0}^{l} \mathbf{N}_{u}^{T} I_{12}\left(\frac{5}{4} \mathbf{N}_{\gamma}-\mathbf{N}_{w, x}\right) d x, \\
& \mathbf{m}_{\gamma \gamma}^{22}=\int_{0}^{l}\left(\frac{5}{4} \mathbf{N}_{\gamma}^{T}-\mathbf{N}_{w, x}^{T}\right) I_{22}\left(\frac{5}{4} \mathbf{N}_{\gamma}-\mathbf{N}_{w, x}\right) d x, \mathbf{m}_{u \gamma}^{34}=-\frac{5}{3 h^{2}} \int_{0}^{l} \mathbf{N}_{u}^{T} I_{34}\left(\mathbf{N}_{\gamma}-\mathbf{N}_{w, x}\right) d x, \\
& \mathbf{m}_{\gamma \gamma}^{44}=-\frac{5}{3 h^{2}} \int_{0}^{l}\left(\frac{5}{4} \mathbf{N}_{\gamma, x}^{T}-\mathbf{N}_{w, x x}^{T}\right) I_{44} \mathbf{N}_{\gamma, x} d x, \mathbf{m}_{\gamma \gamma}^{66}=\frac{25}{9 h^{4}} \int_{0}^{l} \mathbf{N}_{\gamma}^{T} I_{66} \mathbf{N}_{\gamma} d x .
\end{aligned}
$$

Having the element stiffness and mass matrices derived, the equations of motion for the free vibration analysis in the context of finite element analysis can be written in the form

$$
\mathbf{M D}+\mathbf{K D}=\mathbf{0},
$$

where $\mathbf{D}, \mathbf{M}$, and $\mathbf{K}$ are the structural nodal displacement vector, mass and stiffness matrices, obtained by assembling the element displacement vector $\mathbf{d}$, mass matrix $\mathbf{m}$, and stiffness matrix $\mathbf{k}$ over the total elements, respectively. A harmonic response can be assumed, and Eq. (31) leads to

$$
\left(\mathbf{K}-\omega^{2} \mathbf{M}\right) \overline{\mathbf{D}}=\mathbf{0},
$$

where $\omega$ is the circular frequency, $\overline{\mathbf{D}}$ is the vibration amplitude. Eq. (32) leads to an eigenvalue problem, and its solution can be obtained by the standard method.

\section{NUMERICAL RESULTS AND DISCUSSION}

Numerical investigations are carried out in this section to study the effects of the material distribution and temperature rise on the vibration characteristics of the 2-D FGM beam. Otherwise stated, a beam with an aspect ratio $L / h=20$ composed of alumina $\left(\mathrm{Al}_{2} \mathrm{O}_{3}\right)$, zirconia $\left(\mathrm{ZrO}_{2}\right)$, stainless steel (SUS304) and titanium (Ti-6Al-4V) with the material properties given in Ref. [21,22] is employed in the analysis. Alumina, zirconia, steel and titanium are employed as ceramic1, ceramic2, metal1 and metal2, respectively.

Validation and convergence of the derived formulation is firstly confirmed. To this end, Tab. 1 compares the fundamental frequency parameters of a simply supported (SS) 2-D FGM beam in the reference temperature $(\Delta T=0)$ of the present paper with that of Ref. [14], where a finite element formulation based on Timoshenko beam theory was employed. The frequency parameters in Tab. 1 have been obtained for the beam composed of steel (SUS304), aluminum (Al), alumina $\left(\mathrm{Al}_{2} \mathrm{O}_{3}\right)$ and zirconia $\left(\mathrm{ZrO}_{2}\right)$ 
Table 1. Comparison of fundamental frequency parameter of S-S 2-D FGM beam

\begin{tabular}{|c|c|c|c|c|c|c|c|c|c|}
\hline & & 0 & $n_{x}=\frac{1}{3}$ & $n_{x}=\frac{1}{2}$ & $n_{x}=\frac{5}{6}$ & $x=1$ & $n_{x}=\frac{4}{3}$ & $n_{x}=\frac{3}{2}$ & $n_{x}=2$ \\
\hline \multirow[b]{2}{*}{$n_{z}=$} & & & & & & & & & \\
\hline & & & & & & & & & \\
\hline \multirow[b]{2}{*}{$n_{z}=\frac{\overline{3}}{3}$} & & & & & & & & & \\
\hline & & & 0 & & 51 & & & 791 & \\
\hline \multirow[b]{2}{*}{$n_{z}=\frac{1}{2}$} & & & & & & & & & \\
\hline & & & & & 37 & & 05 & 35 & \\
\hline \multirow[b]{2}{*}{$n_{z}=\frac{\overline{6}}{6}$} & & & & & & & & & \\
\hline & & & 96 & 06 & 47 & & 368 & 93 & 46 \\
\hline \multirow[b]{2}{*}{$n_{z}=1$} & & & & & & & & & \\
\hline & Present & 3.0359 & 3.2983 & 3.3818 & 3.5034 & 3.5493 & 3.6217 & 3.6507 & 3.7175 \\
\hline
\end{tabular}

as previously used in [14] and the fundamental frequency parameter is defined as $\bar{\mu}=$ $\omega_{1} \frac{L^{2}}{h} \sqrt{\rho_{\mathrm{Al}} / E_{\mathrm{Al}}}$, where $\omega_{1}$ is the fundamental frequency of the beam. Very good agreement between the result of the present work with that of Ref. [14] is noted from Tab. 1.

In order to verify the formulation in some further, Tab. 2 compares the fundamental frequency parameter of a S-S unidirectional transverse FGM beam in thermal environment with the result based on Euler-Bernoulli beam theory and the differential transform method of Ref. [22]. The beam is formed from SUS304 and $\mathrm{Al}_{2} \mathrm{O}_{3}$ as employed in [22], and the frequency parameter is also defined as $\bar{\mu}=\omega_{1} \frac{L^{2}}{h} \sqrt{\rho_{\text {steel }} / E_{\text {steel }}}$, where $\rho_{\text {steel }}$ and $E_{\text {steel }}$ are the mass density and Young's modulus of the steel at the reference temperature. A slight difference between the result of the present work with that of Ref. [22] is seen from Tab. 2, and this difference may be resulted from the different beam theory employed herein with that of Ref. [22].

Table 2. Comparison of fundamental frequency parameter for S-S unidirectional FGM beam in thermal environment

\begin{tabular}{cccccc}
\hline$\Delta T(\mathrm{~K})$ & Source & $n=0.1$ & $n=0.2$ & $n=0.5$ & $n=1$ \\
\hline \multirow{2}{*}{20} & Ref. [22] & 4.6536 & 4.3867 & 3.8974 & 3.5193 \\
& Present & 4.6080 & 4.3456 & 3.8645 & 3.4923 \\
\hline \multirow{2}{*}{40} & Ref. [22] & 4.4516 & 4.1782 & 3.6779 & 3.2925 \\
& Present & 4.3966 & 4.1286 & 3.6380 & 3.2595 \\
\hline \multirow{2}{*}{80} & Ref. [22] & 4.0148 & 3.7212 & 3.1834 & 2.7693 \\
& Present & 3.9388 & 3.6530 & 3.1290 & 2.7242 \\
\hline
\end{tabular}


The convergence of the derived formulation in evaluating frequency of the 2-D FGM beam is shown in Tab. 3, where the frequency parameters of a S-S 2-D FGM beam in the reference temperature $(\Delta T=0)$ obtained by different number of the derived elements are listed for various values of the grading indexes $n_{z}$ and $n_{x}$. The convergence of the derived formulation, as seen from Tab. 3 , is very fast, and the convergence is achieved by using just fourteen elements, regardless of the grading indexes. The frequency parameter in Tab. 3 and hereafter is defined as follows $\mu_{i}=\omega_{i} \frac{L^{2}}{h} \sqrt{\rho_{0} / E_{0}}$ where $\omega_{i}$ is the $i^{t h}$ natural frequency, $\rho_{0}$ and $E_{0}$ are the mass density and Young's modulus of SUS304 in the reference temperature.

Table 3. Convergence of the formulation in evaluating frequency parameter $\mu_{1}$ of S-S beam $(\Delta T=0)$

\begin{tabular}{ccccccc}
\hline \multirow{2}{*}{ Grading indexes } & \multicolumn{6}{c}{ Number of elements (ne) } \\
\cline { 2 - 7 } & 5 & 10 & 14 & 16 & 18 & 20 \\
\hline$n_{x}=n_{z}=\frac{1}{3}$ & 3.4042 & 3.4037 & 3.4037 & 3.4037 & 3.4037 & 3.4037 \\
$n_{x}=n_{z}=\frac{1}{2}$ & 3.4223 & 3.4217 & 3.4217 & 3.4217 & 3.4217 & 3.4217 \\
$n_{x}=n_{z}=1$ & 3.3943 & 3.3935 & 3.3934 & 3.3934 & 3.3934 & 3.3934 \\
$n_{x}=n_{z}=2$ & 3.3045 & 3.3035 & 3.3034 & 3.3034 & 3.3034 & 3.3034 \\
\hline
\end{tabular}

Tab. 4 lists the fundamental frequency parameter $\mu_{1}$ of the S-S beam for various values of the grading indexes and a temperature rise $\Delta T=20 \mathrm{~K}$. The table shows a significant influence of the grading indexes on the fundamental frequency of the beam. The effect of the grading index $n_{z}$, which governs the variation of the material properties in the thickness direction, is clearly seen from Tab. 4, where the frequency parameter $\mu_{1}$ is decreased by the increase in the index $n_{z}$, irrespective of the index $n_{x}$. A careful examination of the table shows that the decrease of $\mu_{1}$ by increasing $n_{z}$ is more significant for the beam with a higher index $n_{x}$. For example, a decrease of $20.22 \%$ is attained when increasing $n_{z}$ from 0 to 2 for the beam with $n_{x}=0.2$, but the corresponding values are $30.31 \%$ and $34.36 \%$ for the beam associated with $n_{x}=1$ and $n_{x}=2$, respectively. The decrease of $\mu_{1}$ by increasing $n_{z}$ is due to the fact that, as seen from Eq. (3), the effective Young's modulus of the beam with a higher index $n_{z}$ is smaller, and this leads to lower beam rigidities. Noting that the mass moments are also decreased by increasing $n_{z}$ but with the constituent materials adopted herein the decrease of the rigidities is much faster. The effect of the index $n_{x}$ on the fundamental frequency parameter $\mu_{1}$, as seen from Tab. 4 , is different from that of the index $n_{z}$, and $\omega_{1}$ increases with the increase of the $n_{x}$ index. The increase of $\mu_{1}$ by increasing $n_{x}$ is, however more significant for the beam associated with a lower index $n_{z}$. The increase of the parameter $\mu_{1}$ by increasing the index $n_{x}$ is explained by the increase in the beam rigidities as clearly seen from Eq. (17).

The variation of the frequency parameter $\mu_{1}$ with the grading indexes $n_{z}$ and $n_{x}$ of the S-S beam is depicted in Fig. 3 for various values of the temperature rise $\Delta T$. Fig. 4 shows the variation of the first four natural frequency parameters $\mu_{i}(i=1, \ldots, 4)$ with 


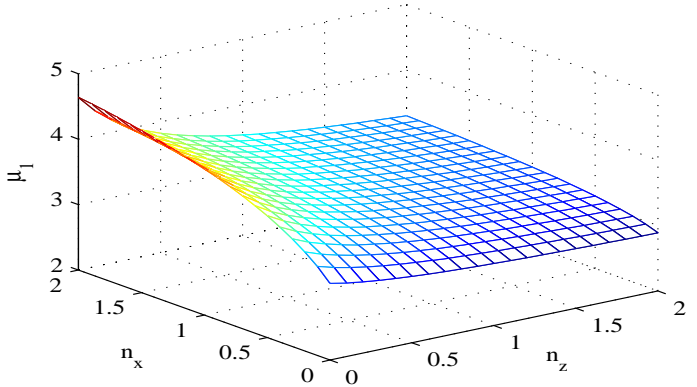

(a) $\Delta \mathrm{T}=0 \mathrm{~K}$

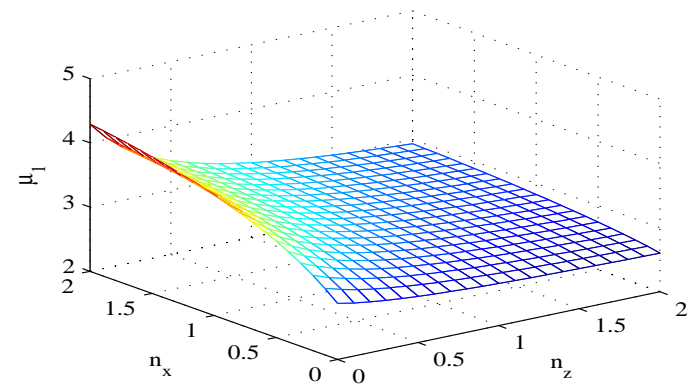

(c) $\Delta \mathrm{T}=40 \mathrm{~K}$

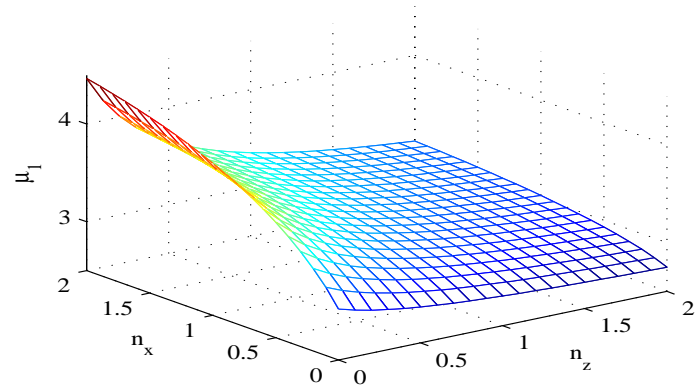

(b) $\Delta \mathrm{T}=20 \mathrm{~K}$

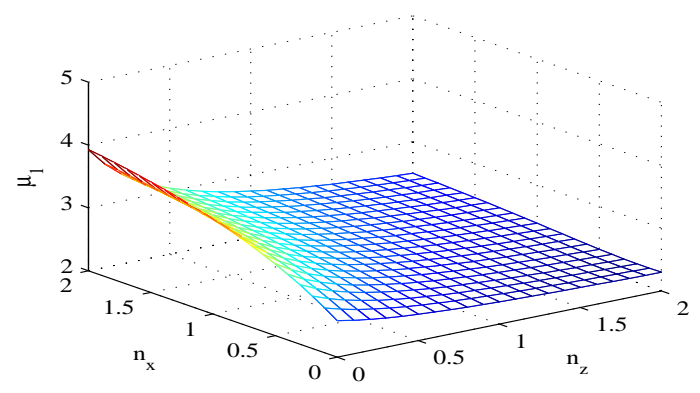

(d) $\Delta \mathrm{T}=80 \mathrm{~K}$

Fig. 3. Variation of frequency parameter $\mu_{1}$ with grading indexes of S-S beam for different $\Delta T$
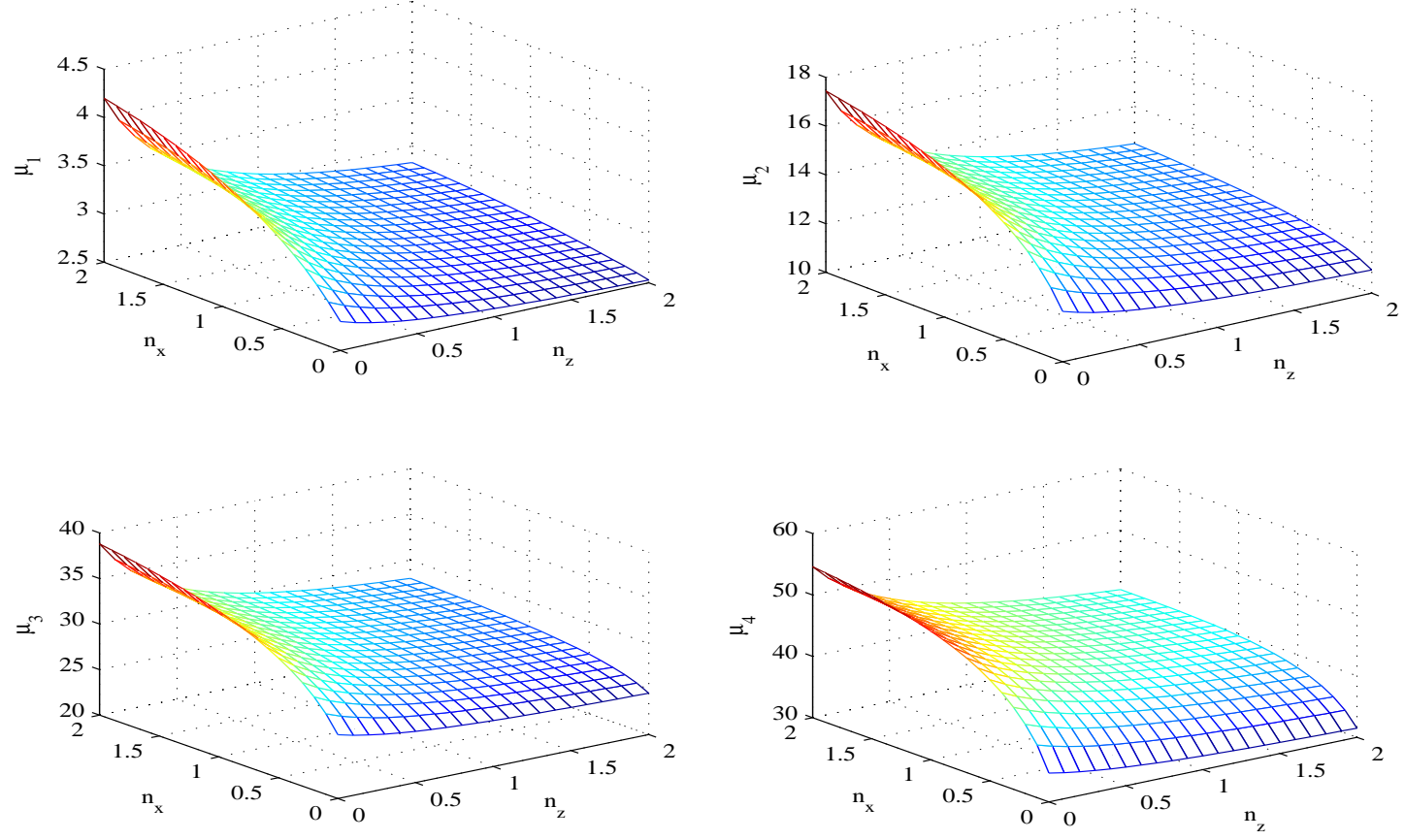

Fig. 4. Variation in the first four natural frequency parameters of S-S beam for $\Delta T=50 \mathrm{~K}$ 


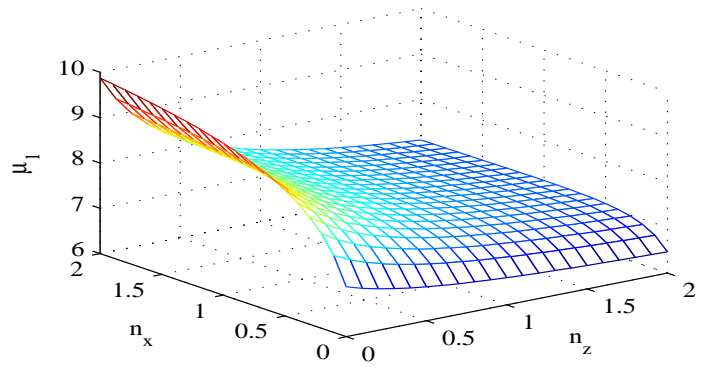

(a) $\Delta \mathrm{T}=0 \mathrm{~K}$

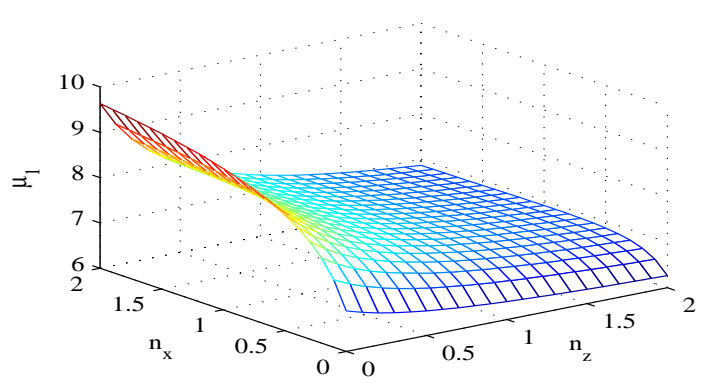

(c) $\Delta \mathrm{T}=40 \mathrm{~K}$

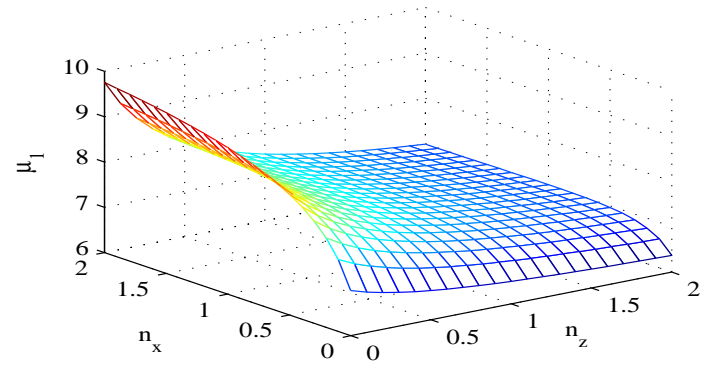

(b) $\Delta \mathrm{T}=20 \mathrm{~K}$

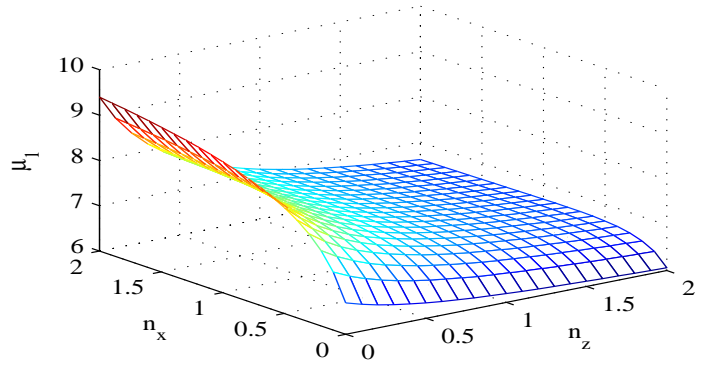

(d) $\Delta \mathrm{T}=80 \mathrm{~K}$

Fig. 5. Variation of frequency parameter $\mu_{1}$ with grading indexes of $\mathrm{C}-\mathrm{C}$ beam for different $\Delta T$
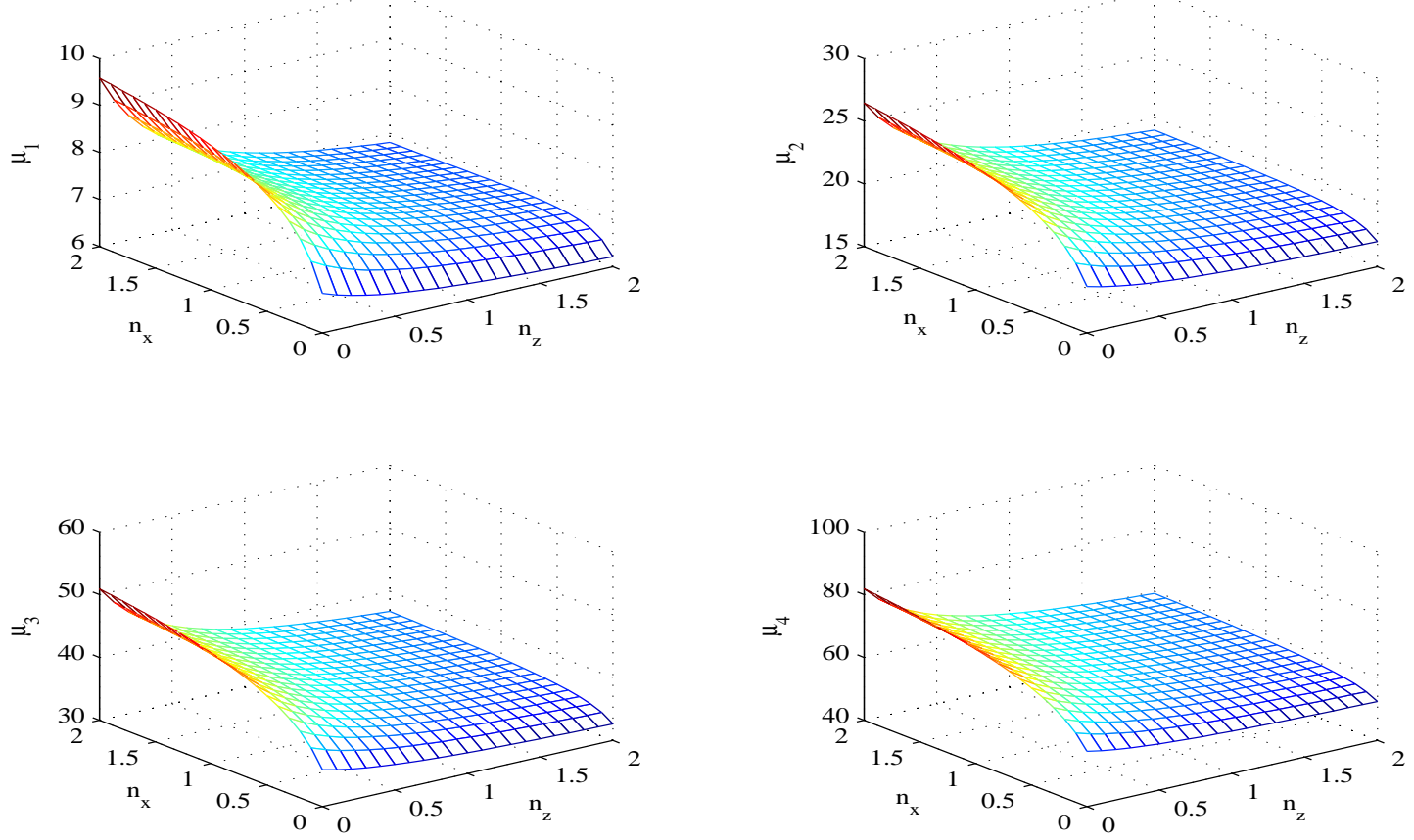

Fig. 6. Variation in the first four natural frequency parameters of $\mathrm{C}-\mathrm{C}$ beam for $\Delta T=50 \mathrm{~K}$ 
Table 4. Fundamental frequency parameter of S-S beam for various grading indexes and $\Delta T=20 \mathrm{~K}$

\begin{tabular}{lccccccc}
\hline & $n_{x}=0$ & $n_{x}=0.2$ & $n_{x}=0.5$ & $n_{x}=1.0$ & $n_{x}=1.2$ & $n_{x}=1.5$ & $n_{x}=2.0$ \\
\hline$n_{z}=0$ & 3.0227 & 3.3456 & 3.6853 & 4.0587 & 4.1671 & 4.3004 & 4.4657 \\
$n_{z}=0.2$ & 2.9415 & 3.1905 & 3.4464 & 3.7213 & 3.7998 & 3.8955 & 4.0129 \\
$n_{z}=0.5$ & 2.8690 & 3.0551 & 3.2422 & 3.4402 & 3.4963 & 3.5643 & 3.6472 \\
$n_{z}=1.0$ & 2.8051 & 2.9394 & 3.0714 & 3.2103 & 3.2496 & 3.2972 & 3.3551 \\
$n_{z}=1.2$ & 2.7890 & 2.9111 & 3.0302 & 3.1556 & 3.1912 & 3.2343 & 3.2867 \\
$n_{z}=1.5$ & 2.7704 & 2.8785 & 2.9833 & 3.0940 & 3.1255 & 3.1637 & 3.2102 \\
$n_{z}=2.0$ & 2.7483 & 2.8406 & 2.9293 & 3.0236 & 3.0506 & 3.0835 & 3.1236 \\
\hline
\end{tabular}

the grading indexes $n_{z}$ and $n_{x}$ of the S-S beam for a temperature rise $\Delta T=50 \mathrm{~K}$. The effect of the grading indexes on the fundamental frequency is clearly seen again from Fig. 3 , where the frequency parameter decreases by increasing the index $n_{z}$, but it increases by increasing the index $n_{x}$, regardless of the temperature rise. The temperature rise leads to a lower frequency, but it hardly changes the variation of the parameter on the grading indexes. The effect of the grading indexes on the higher frequencies, as seen from Fig. 4, is similar to the case of the fundamental frequency, and at the given value of the temperature rise, the frequency parameters $\mu_{2}, \mu_{3}$ and $\mu_{4}$ are also decreased by increasing the index $n_{z}$ and they are increased by increasing index $n_{x}$.

In order to show the effect of boundary conditions on the relation between the grading indexes and frequency parameter, Figs. 5 and 6 illustrate the variation of the fundamental frequency parameter and the first four frequency parameters on the grading indexes of the 2-D FGM beam with clamped ends (C-C beam) in thermal environment, respectively.

The corresponding figures for the beam with one end clamped and the other free (CF) are depicted in Figs. 7 and 8. The effect of the boundary conditions is clearly seen from the figures. As expected, the frequency parameters of the $\mathrm{C}-\mathrm{C}$ beam are much higher than that of the beam with the other boundary conditions, regardless of the grading indexes and the temperature rise. The variation of the frequency parameters with the grading indexes of the C-C beam and the C-F beam is similar to that of the S-S beam, and the frequency parameters decrease when increasing the index $n_{z}$ and they increased with increasing the index $n_{x}$. In addition, the change in the frequency parameters by the variation of the indexes of the C-F beam is more significant than that of the S-S beam and the $\mathrm{C}-\mathrm{C}$ beam. In other words, the $\mathrm{C}-\mathrm{F}$ beam is more sensitive to the change in the grading indexes than the S-S and C-C beams.

In Fig. 9, the mode shapes for $w_{0}, u_{0}$ and $\gamma_{0}$ of the S-S beam are depicted for $\Delta T=0$. For $n_{x}=0$, the beam deduces to the unidirectional FGM beam, and thus Fig. 9(a) represents the mode shapes of the unidirectional transverse beam composed of zirconia and titanium. As can be seen from the figure, the mode shapes of the 2-D FGM beam as depicted in Fig. 9(b) are very different from that of the unidirectional transverse FGM beam. 
The longitudinal variation of the material properties of the 2-D FGM beam significantly influences on the vibration modes. While the first and third modes of the transverse

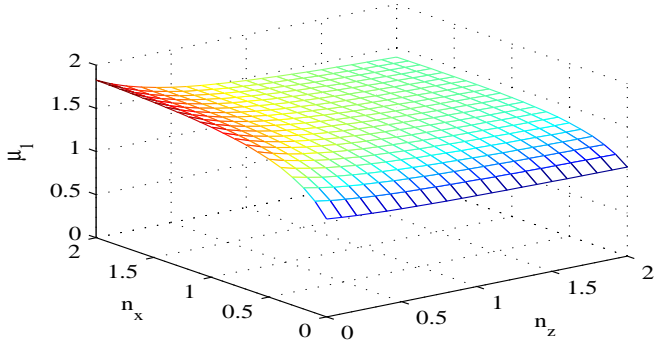

(a) $\Delta \mathrm{T}=0 \mathrm{~K}$

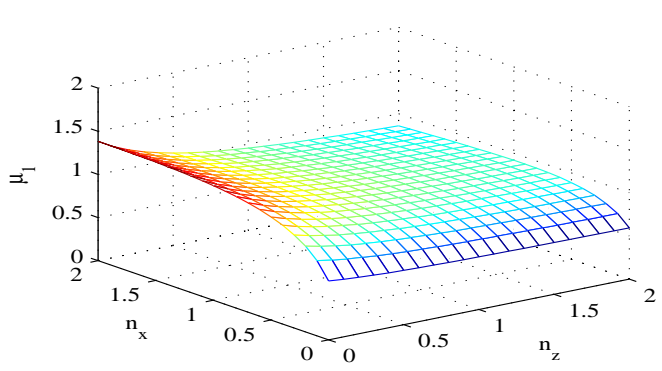

(c) $\Delta \mathrm{T}=40 \mathrm{~K}$

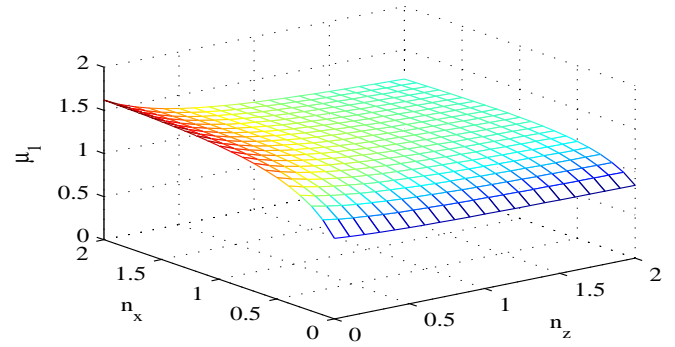

(b) $\Delta \mathrm{T}=20 \mathrm{~K}$

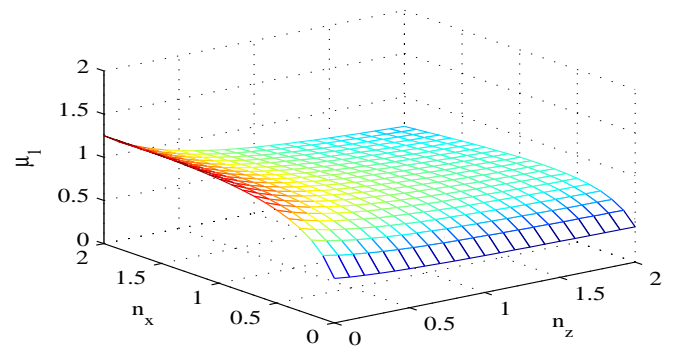

(d) $\Delta \mathrm{T}=50 \mathrm{~K}$

Fig. 7. Variation of frequency parameter $\mu_{1}$ with grading indexes of the C-F beam for different $\Delta T$
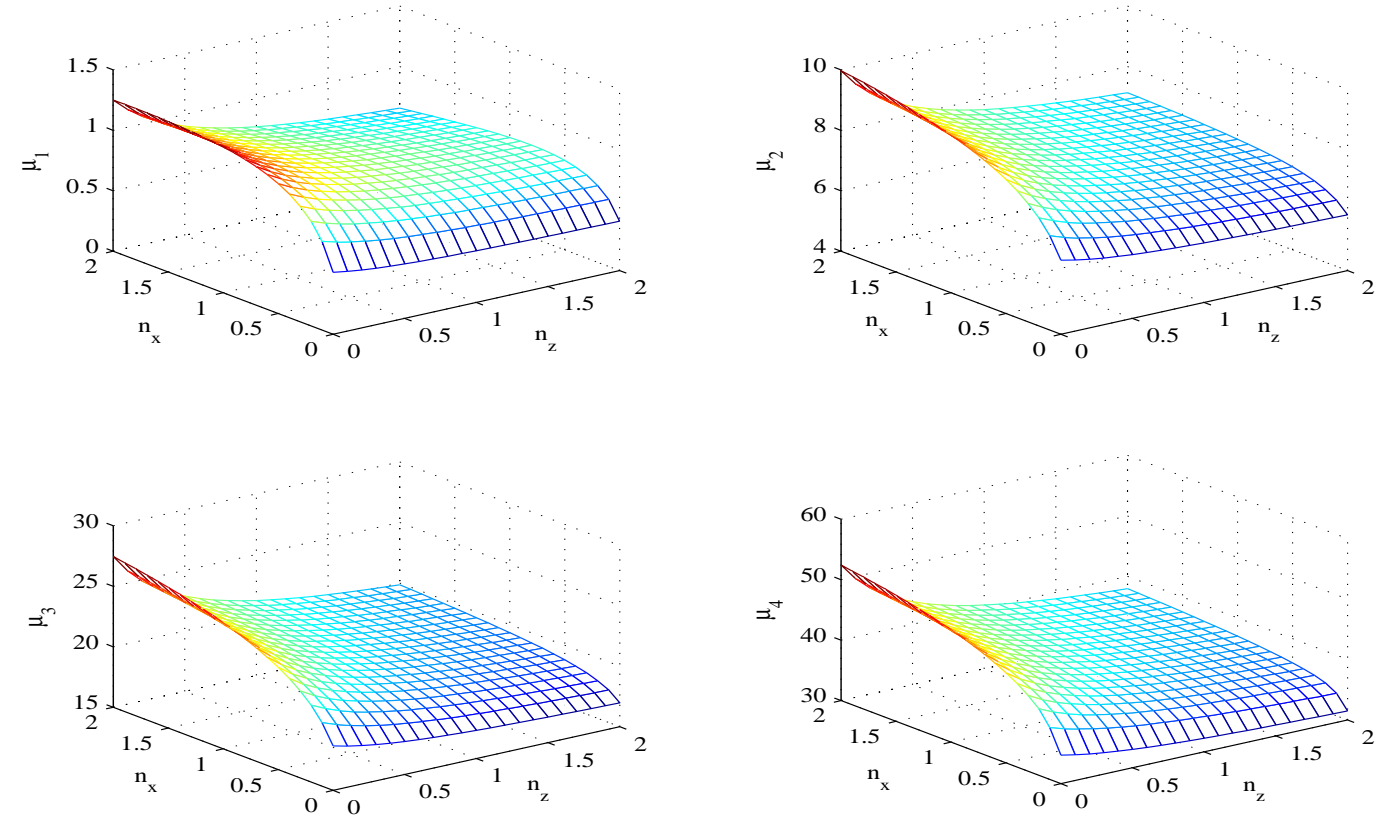

Fig. 8. Variation in the first four natural frequency parameters of $\mathrm{C}-\mathrm{F}$ beam for $\Delta T=50 \mathrm{~K}$ 

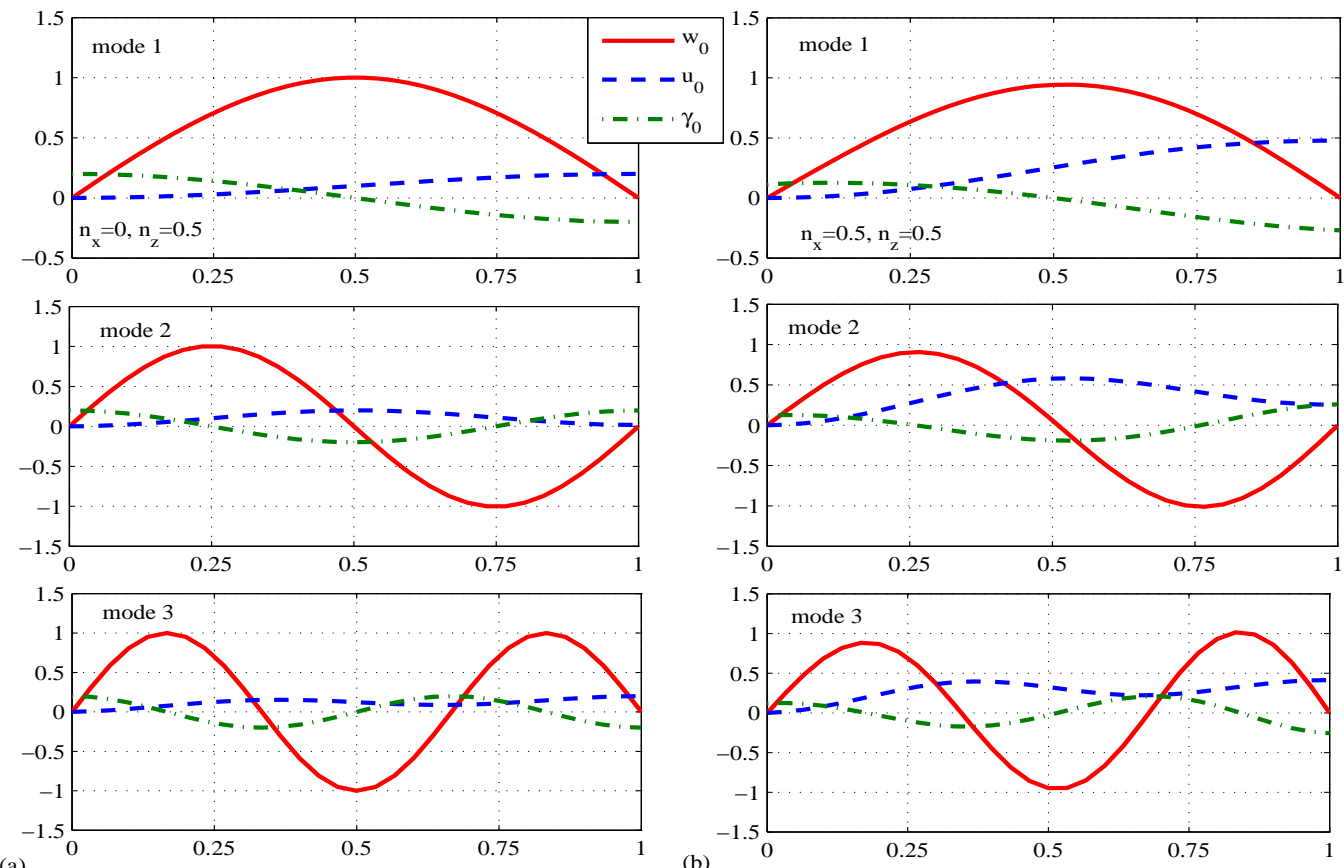

(b)

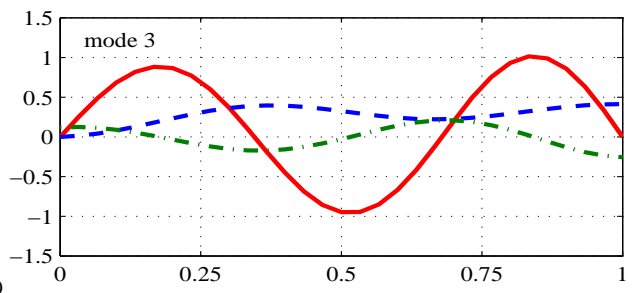

Fig. 9. Vibration mode shapes of S-S beam for $\Delta T=0$ : (a) $n_{x}=0, n_{z}=0.5$; (b) $n_{z}=0.5, n_{x}=0.5$

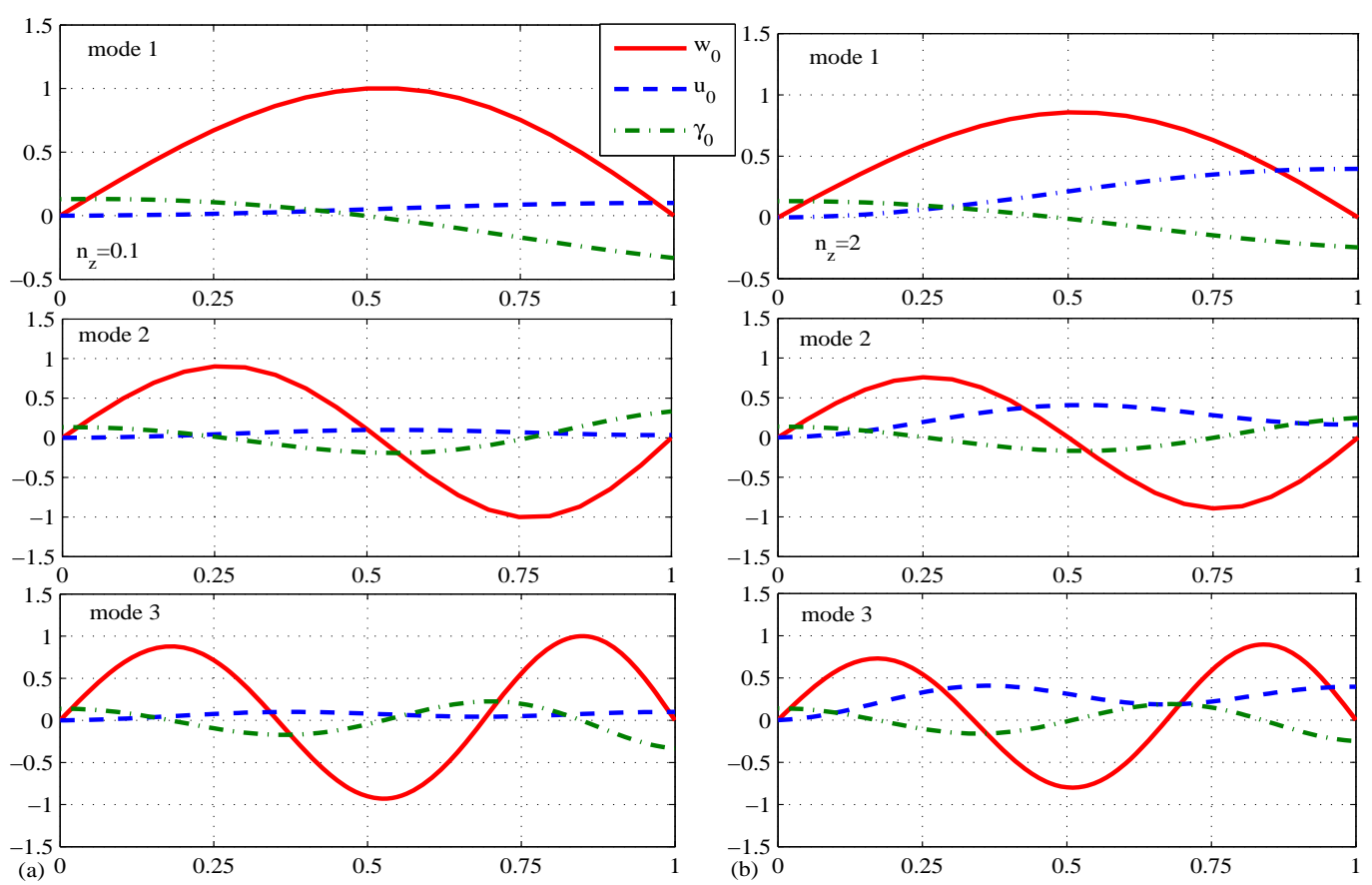

Fig. 10. Vibration mode shapes of S-S beam with $n_{x}=1$ : (a) $n_{z}=0.1$, (b) $n_{z}=2(\Delta T=50 \mathrm{~K})$ 
displacement $w_{0}$ of the unidirectional FGM beam are symmetric with respect to the midspan, that of the 2-D FGM beam are not. The figure also shows the difference in the mode shape of $u_{0}$ and $\gamma_{0}$ of the 2-D FGM beam with that of the unidirectional beam, and the asymmetric of the second mode for $\gamma_{0}$ with respect to the mid-span is clearly seen from Fig. 9(b). The mode shapes for the $w_{0}, u_{0}$ and $\gamma_{0}$ of the S-S 2D-FGM beam in thermal environment are depicted in Figs. 10 and 11 for various values of the grading indexes and for a temperature rise $\Delta T=50 \mathrm{~K}$. As seen from the figures, the grading indexes have a significant influence on the vibration modes of the beam, and not only vibration amplitude but also the position of the critical point is changed by the variation of the grading indexes.
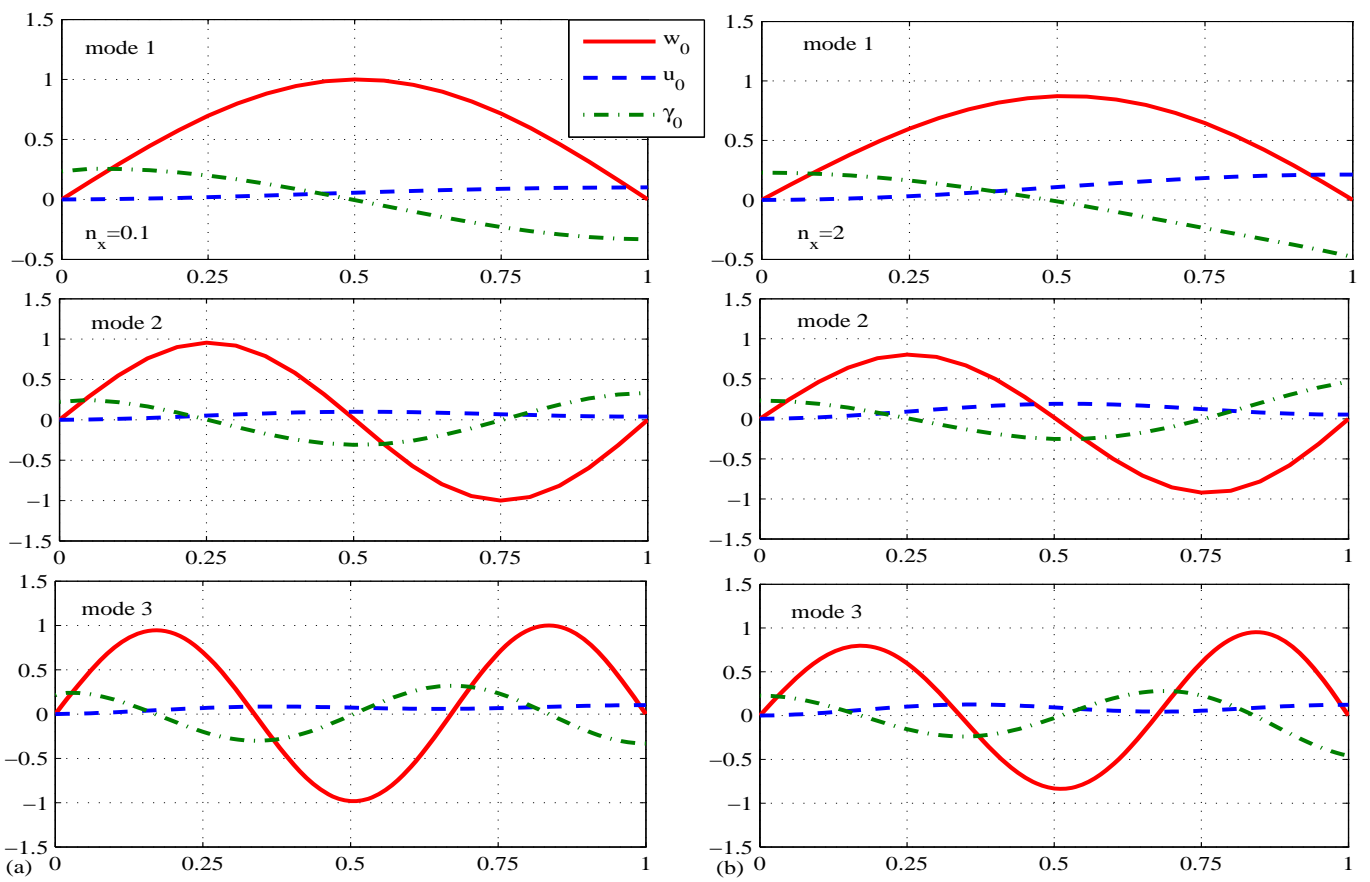

Fig. 11. Vibration mode shapes of S-S beam with $n_{z}=1$ : (a) $n_{x}=0.1$, (b) $n_{x}=2(\Delta T=50 \mathrm{~K})$

The effect of the beam aspect ratio, $L / h$, on the vibration characteristics of the beam is illustrated in Fig. 12, where the variations of the fundamental frequency parameter with the grading indexes of the S-S beam are depicted for two values of the aspect ratio, $L / h=10$ and $L / h=30$, and for a temperature rise $\Delta T=50 \mathrm{~K}$. The aspect ratio, as seen from the figure, has a significant influence on the fundamental frequency parameter of the beam, and as expected an increase in the aspect ratio leads to a decrease of the fundamental frequency parameter, but it hardly changes the relationship between the frequency parameter and the grading indexes. 


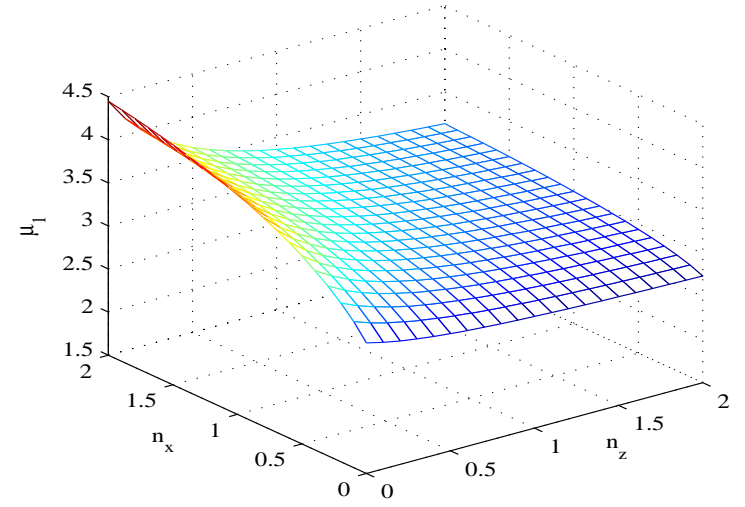

(a) $\Delta \mathrm{T}=50 \mathrm{~K}, \mathrm{~L} / \mathrm{h}=10$

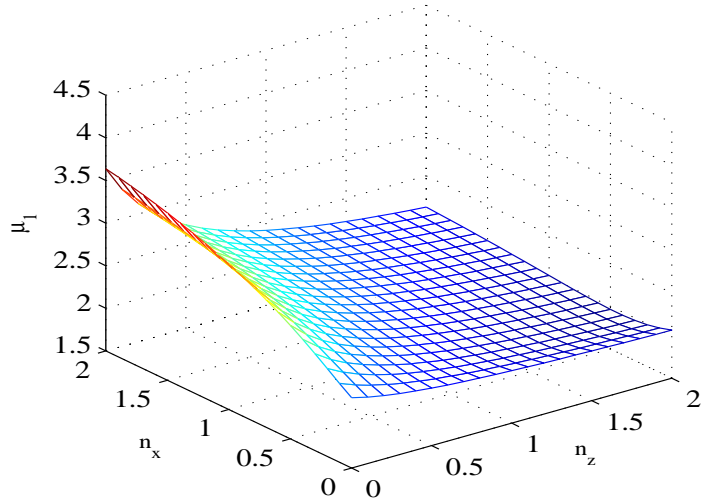

(b) $\Delta \mathrm{T}=50 \mathrm{~K}, \mathrm{~L} / \mathrm{h}=30$

Fig. 12. Variation of fundamental frequency parameter with grading indexes of S-S beam in thermal environment with different values of aspect ratio

\section{CONCLUSION}

The free vibration analysis of 2-D FGM beams in thermal environment by using a new third-order shear deformation theory has been presented. The material properties of the beams are assumed to vary in both the thickness and longitudinal directions and they are considered to be temperature-dependent. The equations of motion in which the transverse shear rotation is employed as an independent variable have been derived from Hamilton's principle and they were solved by a finite element model. The numerical result reveals that the finite element formulation developed in the present work is accurate, and it is capable to give accurate natural frequencies by using a small number of elements. A parametric study has been carried out to highlight the effect of the material grading indexes and the temperature rise on the natural frequencies and mode shapes of the beams. The effect of boundary conditions and the aspect ratio on the vibration characteristics of the beams has also been examined. Though the present paper deals with the free vibration analysis, the finite element formulation derived herein is capable to use for forced vibration analysis of 2-D FGM beams.

\section{ACKNOWLEDGEMENT}

The work presented in this paper has been supported by Vietnam National Foundation for Science and Technology Development (NAFOSTED), Grant No. 107.02-2015.02.

\section{REFERENCES}

[1] A. Chakraborty, S. Gopalakrishnan, and J. N. Reddy. A new beam finite element for the analysis of functionally graded materials. International Journal of Mechanical Sciences, 45, (3), (2003), pp. 519-539. doi:10.1016/s0020-7403(03)00058-4.

[2] R. Kadoli, K. Akhtar, and N. Ganesan. Static analysis of functionally graded beams using higher order shear deformation theory. Applied Mathematical Modelling, 32, (12), (2008), pp. 2509-2525. doi:10.1016/j.apm.2007.09.015. 
[3] S. C. Pradhan and T. Murmu. Thermo-mechanical vibration of FGM sandwich beam under variable elastic foundations using differential quadrature method. Journal of Sound and Vibration, 321, (1), (2009), pp. 342-362. doi:10.1016/j.jsv.2008.09.018.

[4] A. Mahi, E. A. Adda Bedia, A. Tounsi, and I. Mechab. An analytical method for temperature-dependent free vibration analysis of functionally graded beams with general boundary conditions. Composite Structures, 92, (8), (2010), pp. 1877-1887. doi:10.1016/j.compstruct.2010.01.010.

[5] G. Shi. A new simple third-order shear deformation theory of plates. International Journal of Solids and Structures, 44, (13), (2007), pp. 4399-4417. doi:10.1016/j.ijsolstr.2006.11.031.

[6] N. Wattanasakulpong, B. G. Prusty, and D. W. Kelly. Thermal buckling and elastic vibration of third-order shear deformable functionally graded beams. International Journal of Mechanical Sciences, 53, (9), (2011), pp. 734-743. doi:10.1016/j.ijmecsci.2011.06.005.

[7] A. E. Alshorbagy, M. A. Eltaher, and F. F. Mahmoud. Free vibration characteristics of a functionally graded beam by finite element method. Applied Mathematical Modelling, 35, (1), (2011), pp. 412-425. doi:10.1016/j.apm.2010.07.006.

[8] H. T. Thai and T. P. Vo. Bending and free vibration of functionally graded beams using various higher-order shear deformation beam theories. International Journal of Mechanical Sciences, 62, (1), (2012), pp. 57-66. doi:10.1016/j.ijmecsci.2012.05.014.

[9] T. P. Vo, H. T. Thai, T. K. Nguyen, and F. Inam. Static and vibration analysis of functionally graded beams using refined shear deformation theory. Meccanica, 49, (1), (2014), pp. 155-168. doi:10.1007/s11012-013-9780-1.

[10] T. K. Nguyen, T. P. Vo, and H. T. Thai. Static and free vibration of axially loaded functionally graded beams based on the first-order shear deformation theory. Composites Part B: Engineering, 55, (2013), pp. 147-157. doi:10.1016/j.compositesb.2013.06.011.

[11] L. C. Trinh, T. P. Vo, H. T. Thai, and T. K. Nguyen. An analytical method for the vibration and buckling of functionally graded beams under mechanical and thermal loads. Composites Part B: Engineering, 100, (2016), pp. 152-163. doi:10.1016/j.compositesb.2016.06.067.

[12] Z. H. Wang, X. H. Wang, G. D. Xu, S. Cheng, and T. Zeng. Free vibration of twodirectional functionally graded beams. Composite Structures, 135, (2016), pp. 191-198. doi:10.1016/j.compstruct.2015.09.013.

[13] M. Şimşek. Bi-directional functionally graded materials (BDFGMs) for free and forced vibration of Timoshenko beams with various boundary conditions. Composite Structures, 133, (2015), pp. 968-978. doi:10.1016/j.compstruct.2015.08.021.

[14] D. K. Nguyen, Q. H. Nguyen, T. T. Tran, and V. T. Bui. Vibration of bi-dimensional functionally graded timoshenko beams excited by a moving load. Acta Mechanica, 228, (1), (2017), pp. 141-155. doi:10.1007/s00707-016-1705-3.

[15] A. Karamanl1. Bending behaviour of two directional functionally graded sandwich beams by using a quasi-3d shear deformation theory. Composite Structures, 174, (2017), pp. 70-86. doi:10.1016/j.compstruct.2017.04.046.

[16] T. V. Do, D. K. Nguyen, N. D. Duc, D. H. Doan, and T. Q. Bui. Analysis of bi-directional functionally graded plates by FEM and a new third-order shear deformation plate theory. Thin-Walled Structures, 119, (2017), pp. 687-699. doi:10.1016/j.tws.2017.07.022.

[17] T. K. Nguyen, K. Sab, and G. Bonnet. First-order shear deformation plate models for functionally graded materials. Composite Structures, 83, (1), (2008), pp. 25-36. doi:10.1016/j.compstruct.2007.03.004. 
[18] G. Shi, K. Y. Lam, and T. E. Tay. On efficient finite element modeling of composite beams and plates using higher-order theories and an accurate composite beam element. Composite Structures, 41, (2), (1998), pp. 159-165. doi:10.1016/s0263-8223(98)00050-6.

[19] G. Shi and K. Y. Lam. Finite element vibration analysis of composite beams based on higher-order beam theory. Journal of Sound and Vibration, 219, (4), (1999), pp. 707-721. doi:10.1006/jsvi.1998.1903.

[20] Y. S. Touloukian. Thermophysical properties of high temperature solid materials. New York: MacMillan, (1967).

[21] Y. W. Kim. Temperature dependent vibration analysis of functionally graded rectangular plates. Journal of Sound and Vibration, 284, (3), (2005), pp. 531-549. doi:10.1016/j.jsv.2004.06.043.

[22] F. Ebrahimi, F. Ghasemi, and E. Salari. Investigating thermal effects on vibration behavior of temperature-dependent compositionally graded euler beams with porosities. Meccanica, 51, (1), (2016), pp. 223-249. doi:10.1007/s11012-015-0208-y. 